
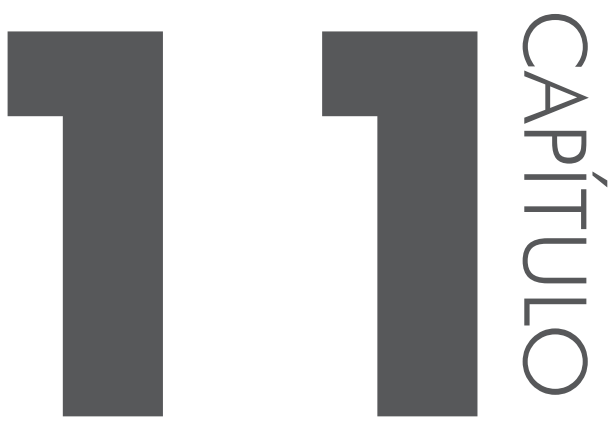

\title{
ESTUDOS SOBRE A COLABORAÇÃO E A INTERACÃÓ EM DIFERENTES CONTEXTOS DE ENSINO E APRENDIZAGEM DE LÍNGUAS NO AMBIENTE DIGITAL
}

Dánie Marcelo de Jesus Universidade Federal do Mato Grosso

Francisco José Quaresma de Figueiredo Universidade Federal de Goiás

Andrea da Silva Marques Ribeiro Universidade do Estado do Rio de Janeiro

\subsection{INTRODUC̣ÃO}

Nos últimos anos, o crescente aumento das relações sociais no ambiente digital gerou espaços de aprendizagem que atraíram pesquisadores interessados na 
potencialidade da interação e do processo de colaboração em contexto de aprendizagem virtual (BEDRAN; SALOMÃO, 2013; DILLENBOURG, 1999; JESUS, 2014; JESUS; MACIEL, 2015; LEFFA, 2003; MARTINO, 2015; TELLES, 2009). Isso se deu em decorrência da pressão social advinda principalmente da realidade global e das tecnologias digitais de informações (TDICs).

As instituições educacionais, por sua vez, começaram a valorizar as TDICs tanto na educação básica quanto em cursos de formação continuada ou pré-serviço de professores. O ambiente acadêmico tem inserido múltiplos recursos digitais, tais como os blogs (GONÇALVES, 2009; FIGUEIREDO; GONÇALVES, 2015), os chats e os fóruns de discussão (JESUS, 2007; RIBEIRO, 2010; SOUZA, 2002) e projetos telecolaborativos (SOUZA, 2003; VASALLO; TELLES, 2006, 2009), que têm como desafio renovar práticas escolares que tornem o processo de ensino mediado pelo computador mais colaborativo e eficiente.

Este capítulo objetiva compreender processos de colaboração em três estudos desenvolvidos em diferentes contextos brasileiros. Os exemplos apresentados são recortes de uma tese de doutorado (SILVA, 2012) e de uma dissertação de mestrado (BRESOLIN, 2011), orientadas por dois dos autores deste artigo, associadas à pesquisa desenvolvida pela terceira autora deste trabalho (RIBEIRO, 2010). Essas investigações fazem parte de uma série de trabalhos de caráter interpretativo a respeito do ensino e da aprendizagem de línguas em contexto virtual, cujo foco é entender como as práticas ciberespaciais podem criar condições para a construção de um processo mais colaborativo de ensino e aprendizagem de línguas sob a égide de interações mediadas pelo computador. Dos resultados, espera-se colher subsídios que possam auxiliar na discussão da formação de educadores na seara digital.

Procuramos, então, estudar as interações entre os participantes, com a finalidade de entender como a colaboração se deu nesses momentos interacionais. Para dar conta dessa empreitada, apresentamos, inicialmente, questões teóricas relacionadas com a teoria sociocultural, bem como com o ensino e com a aprendizagem colaborativa de línguas estrangeiras. Em um segundo momento, discutimos alguns dados dos três estudos, explicitando o contexto e seus participantes. Finalmente, explanamos algumas implicações para o ensino de línguas e as reflexões a que chegamos com esta investigação.

\subsection{TEORIA SOCIOCULTURAL, INTERAC̣ÃO E COLABORAC̣ÃO NO ENSINO E APRENDIZAGEM DE LÍNGUAŚ}

A teoria sociocultural - baseada principalmente nos trabalhos de L. S. Vygotsky e seus colaboradores - postula que o desenvolvimento psicológico da 
criança ocorre por meio da interação com crianças mais experientes e/ou com adultos. Segundo o autor,

qualquer função no desenvolvimento cultural da criança aparece duas vezes, ou em dois planos. Primeiro, ele aparece no plano social e, posteriormente, no plano psicológico. Primeiro, ele aparece entre as pessoas como uma categoria interpsicológica, e, posteriormente, na criança como uma categoria intrapsicológica” (VYGOTSKY, 1981, p. 163).

O desenvolvimento cognitivo é favorecido por estruturas de apoio conhecidas como scaffolding ${ }^{1}$ e ocorre na zona de desenvolvimento proximal (ZDP). A ZDP é definida como "a distância entre o nível de desenvolvimento real, que se costuma determinar através da solução independente de problemas, e o nível de desenvolvimento potencial, determinado através da solução de problemas sob a orientação de um adulto ou em colaboração com companheiros mais capazes” (VYGOTSKY, 1998, p. 112).

Ou seja, a ZDP é a diferença entre o que a criança é capaz de fazer quando age sozinha e o que é capaz de fazer com o auxílio de alguém mais experiente.

Com base na teoria sociocultural, podemos perceber a importância do papel da interação no processo de aprendizagem, visto que "o conhecimento é coconstruído e a aprendizagem sempre envolve mais do que uma pessoa" (NYIKOS; HASHIMOTO, 1997, p. 507). Na sala de aula de línguas, seja de língua materna (L1), seja de segunda língua (L2), a interação ainda é mais importante, pois a língua é tanto o objeto de conhecimento quanto o meio para a aprendizagem (TSUI, 1995).

Coelho (1992, p. 37) afirma que a aquisição de L2 é favorecida quando proporcionamos aos alunos "oportunidades para interação frequente e extensa na língua-alvo", oportunidades essas que são favorecidas por um modelo de ensino que promova a aprendizagem colaborativa. Esta consiste em uma abordagem em que duas ou mais pessoas aprendem ou tentam aprender algo juntas, seja por meio de interações em sala de aula ou fora dela, seja por interações mediadas pelo computador, cuja ênfase recai na coconstrução do conhecimento.

Essa abordagem tem sido objeto de estudo de vários pesquisadores (BRUFFEE, 1999; DILLENBOURG, 1999; DONATO, 2004; FIGUEIREDO, 2005, 2006; LIANG; MOHAN; EARLY, 1998; LIMA, 2011; NUNES, 2002, entre outros) devido ao fato de que a interação não ajuda apenas os alunos menos experientes: ela

1 Wood, Bruner e Ross (1976) cunharam o termo scaffolding como uma metáfora para descrever o apoio dado pelas mães às suas crianças quando as ajudavam a fazer uma construção piramidal com um conjunto de blocos de madeiras, durante um experimento. É descrito como um processo que possibilita à criança ou ao aprendiz solucionar um problema, realizar uma tarefa, ou atingir um objetivo que estaria além de seus esforços, caso não tivesse a ajuda de outra pessoa. 
leva também os alunos mais experientes a descobrir novas formas de aprender. Trabalhando juntos, os alunos não compartilham apenas ideias e informações, mas também estratégias de aprendizagem (DONALDSON, 1990; SWAIN, 2000).

Vários autores têm comprovado que, por meio do computador e da internet, a aprendizagem de línguas pode ocorrer além das paredes da sala de aula (LEFFA, 2003; PAIVA, 2001a, 2001b; SOUZA, 2002; WARSCHAUER, 1997, entre outros). Warschauer (1997) advoga que a interação mediada pelo computador pode ser percebida como oportunidade para a socialização, para a produção linguística e para a aprendizagem. Algumas vantagens do meio eletrônico são, por exemplo, o rompimento de limitações espaciais e temporais e o acesso a um grande número de participantes para interagir, o que faz que a aprendizagem seja favorecida pelas trocas interacionais e pela colaboração entre os participantes.

Projetos telecolaborativos têm sido usados também para favorecer a aprendizagem de línguas (cf. DEBSKI, 2001; FIGUEIREDO; SILVA, 2014, 2015; LELOUP; PONTERIO, 2003; SILVA; FIGUEIREDO, 2012; SOUZA, 2003; VASSALLO; TELLES, 2006, 2009). Por meio de tais projetos, alunos de diferentes instituições e de diferentes países, além de se ajudarem na aprendizagem de línguas, podem ter acesso a aspectos relativos a diferenças culturais, bem como aprender diferentes formas de dizer alguma coisa; ou seja, podem desenvolver a sua competência linguístico-comunicativa.

Apesar da ampliação dessas pesquisas que tocam na questão da interação e de processos colaborativos em ambientes digitais, percebe-se, ainda, pouca bibliografia dentro desse tema que descreva contextos de aprendizagem de línguas estrangeiras no Brasil. Assim, acreditamos na necessidade de ampliação desse escopo de investigação com diferentes estudos em contextos diversos. Daí nosso interesse de compartilhar, com os leitores, nossas investigações.

\subsection{ANÁLISE E DISCUSSÃO DOS DADOS}

Com base na análise dos dados coletados em três estudos brasileiros - Silva (2012), Ribeiro (2010) e Bresolin (2011) -, procuramos compreender a construção de processos colaborativos no discurso de alunos e de professores durante atividades interativas mediadas pelo computador. Assim, foi possível depreender amostras representativas desses processos em interação entre professores e alunos, bem como entre alunos, como serão apresentadas.

\section{1.3.1 Primeiro estudo}

O estudo realizado por Silva (2012) sobre o uso da telecolaboração em aulas de Inglês Instrumental tem como contexto dois ambientes: um presencial, outro 
virtual. Em relação ao contexto presencial, participaram dessa pesquisa três instituições de ensino: o IFG e a universidades de Trier e a Universidade de Ciências Aplicadas de Worms, ambas na Alemanha.

Por sua vez, o contexto virtual se refere ao espaço interativo proporcionado pelo uso do aplicativo Openmeetings, um software de web conferência, síncrono e gratuito. Trata-se de um aplicativo que permite conferência por meio de protocolo web, com o uso de um simples navegador, como Internet Explorer, Firefox ou Google Chrome. Esse aplicativo é integrado à plataforma Moodle, portanto o acesso se dá exatamente da mesma forma como ocorre para um fórum, um chat ou qualquer outro recurso dessa plataforma. O Openmeetings permite teleconferência com os seguintes componentes e funções integrados: gerenciamento de permissões; compartilhamento de tela; gravação de áudio e de vídeo; gravação de apresentação; chat; lousa interativa; e apresentação de arquivos ao mesmo tempo em que os usuários interagem através de chat, áudio, vídeo e lousa, entre outros. ${ }^{2}$

O estudo de Silva (2012) buscou verificar como os interagentes trabalham com a língua portuguesa e a língua inglesa nas interações em teletandem. ${ }^{3}$ Participaram do estudo uma professora e três alunos do Curso Superior Tecnológico em Turismo do IFG, três alunos da Universidade de Worms e um aluno da Universidade de Trier. Inicialmente, esses participantes interagiram virtualmente, por meio do Openmeetings, no segundo semestre de 2010 e, posteriormente, os participantes alemães vieram fazer uma visita ao IFG, quando puderam interagir face a face com seus pares.

Como fontes de dados para o estudo, a pesquisadora fez uso de aplicativos computacionais (interações e e-mails), notas de campo, questionário, entrevista e comentários dos participantes, bem como produções acadêmicas feitas pelos participantes do estudo (abstract, resumo e artigo científico).

Ao analisar as interações realizadas entre os alunos brasileiros e alemães, a pesquisadora observou que a língua inglesa foi utilizada com três propósitos: para a aprendizagem da própria língua inglesa, para a aprendizagem do português e para a aprendizagem da língua alemã.

No exemplo a seguir, podemos observar Natalie (participante brasileira) pedindo ajuda a Teego (participante alemão) sobre como dizer a palavra 'verificar' em inglês:

2 Para mais detalhes sobre o aplicativo, veja < https://code.google.com/p/openmeetings/>.

3 A natureza colaborativa da aprendizagem em tandem se torna explícita na própria metáfora que lhe dá nome, uma vez que tandem é a palavra inglesa para bicicletas de dois assentos, ou seja, nas quais o esforço conjunto de dois ciclistas produz o movimento (Souza, 2003, p. 29, n. 4). 
Natalie: No, ok, and then I have that I can to, to, to show for you the photos about our cities in this program Openmeetings, I will, I will pass, I will see 'verificate', 'verificate'? Exists this word? This word 'verificar'?

Teego: Verify.

Natalie: $\quad$ Oh, yes, 'verify'. You have to learn me English too, yes? Because you learn much, much more than me.

Teego: No problem.

Natalie: $\quad$ Yes? So I want that you correct me when I'm wrong.

Teego: $\quad$ Yep, but often you are not wrong, like, only a couple of, like, only a few times and then I will correct you, of course.

Natalie: $\quad$ Yes, you can correct me. So what is the word, 'verific'?

Teego: 'Verify'.

Natalie: $\quad$ 'Verify'. So I will verific, verify, yes, I will verify, yes?

Teego: 'Verify'.

Natalie: $\quad$ Yes, thank you. I will verify about this possibility for you, I will show to you the photos and if it is possible. I will show to you.

Nesse recorte, enquanto falava sobre as cidades turísticas que visitariam em Goiás, Natalie tem a ideia de enviar a Teego, via Openmeetings, algumas fotos desses lugares. Ao se comunicar com o parceiro, Natalie percebe a necessidade de saber como falar em inglês a palavra 'verificar'. Ela, então, hipotetiza que 'verificate' é o termo apropriado. Para confirmar essa hipótese, Natalie faz uso da língua portuguesa dizendo "verificar" como um scaffolding para se certificar, por meio da interação com Teego, se sua hipótese era verdadeira ou não. Teego, então, auxilia sua parceira provendo-lhe a forma "verify" em inglês.

Já no exemplo a seguir, é Natalie que assume o papel de auxiliar e ensina a Teego a pronunciar algumas palavras em português:

Natalie: $\quad$ Yes, yes, it is. You want to learn how pronounce the name of the relatives in Portuguese?

Teego: $\quad$ Yes, I would like to, yeah.

Natalie: $\quad$ Yes. Mother is mãe.

Natalie: [A aluna usa o chat para escrever a palavra] Mãe

Teego: How do you pronounce it?

Natalie: $\quad$ Mãe.

Teego: $\quad$ Mãe. Mãe, mãe, mãe.

Natalie: Or if you want to, like me, I pronounce like a... some child pronounce like this, but is a very... with love, very carefully way to say: mamãe.

Teego: Mamãe. 
Podemos perceber, nesse recorte, que é Natalie quem desempenha um papel mais colaborador no processo interacional, auxiliando Teego, por meio da língua inglesa, na aprendizagem de palavras em português referentes aos membros da família.

$\mathrm{Na}$ sequência, é Teego que aproveita a oportunidade da interação para auxiliar Natalie na aprendizagem de sua língua materna (o alemão). Como a língua alemã não era conhecida por Natalie, Teego usa a língua inglesa como um scaffolding para ir, aos poucos, ensinando a Natalie a pronunciar corretamente a palavra "irmã" em alemão.

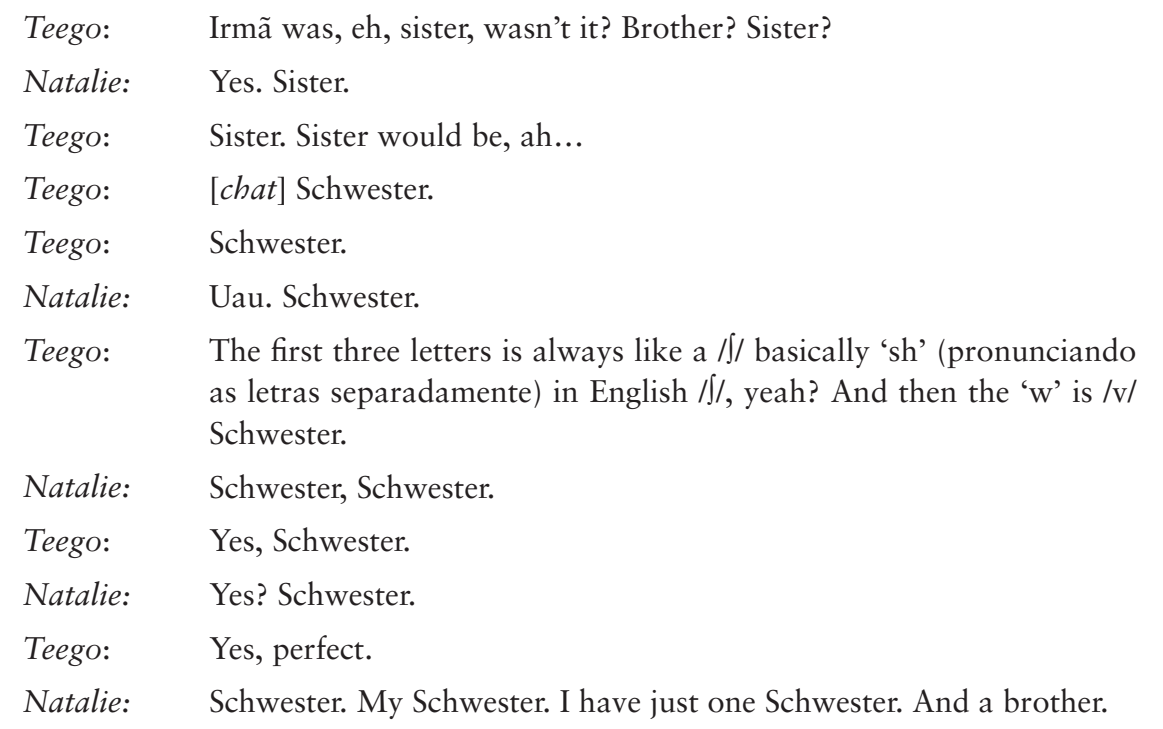

Como podemos perceber, a interação proporcionada pela utilização do aplicativo Openmeetings fez com que os alunos tivessem a oportunidade de escrever e de falar nas línguas que estavam aprendendo. $\mathrm{O}$ uso da teleaprendizagem se configurou, pois, como um recurso a mais que pode ser utilizado nas aulas de Inglês Instrumental, como observado por Natalie, durante uma entrevista:

Natalie: $\quad$ No começo, foi meio complicado porque ele não sabia o sistema. Então, eu percebi que eu tinha mais conhecimento do sistema, do Openmeetings, pelo treinamento, pelos encontros que a gente tinha tido aqui no Brasil, com o nosso grupo do que eles lá na Alemanha. Então, no começo, até a gente pegar o jeito, como mexia, principalmente, para conciliar os horários do Brasil e Alemanha foi mais complicado sim, mas foi uma ferramenta muito boa e de fato contribuiu muito para nosso aprendizado porque, por meio dele, a gente mostrava fotografia, a gente escrevia, no caso, a gente via um ao outro. Então, facilitava muito as interações e o aprendizado também. 
É necessário esclarecer que Natalie e Teego não possuíam conhecimento prévio das línguas que estavam aprendendo por meio das interações: língua alemã e língua portuguesa, respectivamente. Logo, o uso da língua inglesa como língua de ancoragem (scaffolding) para o trabalho com as outras duas línguas foi o meio encontrado pelo par para coconstruir o processo de aprendizagem dessas línguas.

Projetos colaborativos, como postulam Warschauer (1997) e Souza (2003), são uma forma de aprendizagem em que os aprendizes trabalham colaborativamente para aprimorar as habilidades linguísticas, conhecer melhor o outro e sua cultura, e, em algumas parcerias, também para compartilhar conhecimento sobre sua vida profissional. Desse modo, as interações entre Natalie e Teego demonstraram que eles buscaram compartilhar conhecimento linguístico, conhecer detalhes da vida pessoal, profissional e acadêmica um do outro, auxiliando-se, mutuamente, na aprendizagem de línguas que lhes foi proporcionada pelo advento da telecolaboração.

\section{1.3.2 Segundo estudo}

O segundo estudo trata da construção coletiva de ações de suporte ao longo de um curso para professores de língua inglesa, desenvolvido por Ribeiro (2010). Segundo a autora, as ações de suporte são aquelas que configuram "todo o tipo de ajuda oferecido para a solução de problemas, dificuldades e questões de diferentes naturezas (técnica, pedagógica, pessoal etc.) trazidas pelos alunos durante a realização do curso e no âmbito do ambiente virtual” (RIBEIRO, 2010, p. 91). Nesse escopo, a aprendizagem colaborativa no meio digital vai além do conteúdo pedagógico e linguístico. $\mathrm{O}$ estudo destaca que ações de suporte, nem sempre voltadas para a aprendizagem de conteúdo, mostram-se fundamentais para o estabelecimento de relações e também para o processo de ensino e aprendizagem em um espectro mais amplo, típico de um ambiente digital, contribuindo para a consolidação do grupo. O contraponto em relação ao estudo anteriormente apresentado reside na discussão de que a colaboração vai além da interação e do auxílio proporcionado pelos interagentes para a aquisição e o desenvolvimento de conteúdo linguístico. A colaboração mostra-se, por igual, fundamental no desenvolvimento de conhecimentos técnico, de navegação e na solução de dificuldades e de problemas relacionados com a compreensão e a realização das tarefas pedagógicas, com o design do curso e com a superação de problemas de cunho pessoal, que podem surgir em cursos online. Nesse sentido, a colaboração se concretiza em ações de suporte, muitas vezes não planejadas, fomentando, assim, o estabelecimento de relações entre os participantes de tais cursos e a consequente consolidação do grupo.

Os dados foram coletados em uma turma do curso Teacher's links: reflexão e desenvolvimento para professores de inglês, da qual participaram catorze pro- 
fessores. O curso - oferecido pelo Programa em Linguística Aplicada e Estudos da Linguagem (Lael) da Pontifícia Universidade Católica de São Paulo (PUC-SP), com apoio logístico e administrativo da Coordenadoria de Especialização, Aperfeiçoamento e Extensão (Cogeae) e em associação com a Cultura Inglesa de São Paulo - é organizado em três módulos compostos por dois componentes: Reflexão e Desenvolvimento, sendo cada componente mediado por um professor-tutor, ou seja, dois professores-tutores são responsáveis por cada turma. A colaboração, dessa forma, mostrou-se essencial para a construção de ações de suporte para a solução de problemas.

O estudar online é uma atividade bastante complexa, composta de muitos desafios em diversas esferas. Participantes desse contexto enfrentam diferentes tipos de problema ao longo do curso. Segundo Ribeiro (2010) e Carelli (2003), participantes dessa modalidade de ensino relatam problemas de cunho tecnológico e pessoais, dificuldades na realização das tarefas pedagógicas, problemas no gerenciamento de tempo, na compreensão do design do curso, bem como na realização de tarefas em grupo. Relatam, também, problemas relacionados com a falta de proficiência na língua estrangeira usada nos cursos e dificuldades em lidar com questões administrativas.

Essa variada gama de problemas e questões denota a necessidade de que os professores desenvolvam conhecimentos que vão além das questões epistemológicas, pedagógicas e técnicas. Faz-se necessário, então, desenvolver um olhar crítico e sensível para o que ocorre no curso e, assim, construir ações mais adequadas e voltadas não só para aprendizagem e conteúdo do curso, mas também para o estabelecimento de relações entre os participantes e a consequente consolidação da turma na qualidade de grupo.

Neste estudo, foi possível verificar como a colaboração entre os participantes do curso foi fundamental para construção de ações de suporte e no estabelecimento de relações, extrapolando a esfera epistemológica e pedagógica.

A mensagem do aluno Rivaldo postada no fórum Coffee break ${ }^{4}$ é bastante ilustrativa dos tipos de problemas relatados no ambiente do curso.

I think what I can say here is a summary of what I said in the other items, so, be patient and read it!

This opportunity we have now is something everyone has ever not only wanted but needed and I am very glad about that. Nowadays teachers work the whole day and this way it is impossible for me to think about a course in a different standard.

4 O Fórum Coffee Break é um espaço de interação no qual os alunos podem conversar sobres suas vidas pessoais e assuntos fora do escopo do conteúdo do curso. 
I have never done an on line course so it has been a little difficult to deal with this new language (yesterday I did not get to send a message about Paulo Freire, fortunately, today it is OK)

When I look at the screen I am afraid of so many things to read, I get a little confused, it is the stress of our profession that sometimes can almost block our mind, forgive me and help me, please!

Despite this course we have now, it is very difficult for a teacher to have opportunity to talk, read and write in English, even when we are with our colleagues once many of us feel ourselves insecure. I think it is wrong to act this way; to talk, read and write in English is a must for teachers at least when we are at school once when we leave it we can not do it anymore so, instead of complaining, let us try it hard. I think some are afraid of the false cognates, many words with double letters, grammar, and the judgment a "colleague" can do and also the colleagues we studied in have not prepared most of us properly so, it is our obligation to go after it!

Sometimes I am afraid of the "correct size" a text typed here should be not to make it too long, so, let me stop it now!

(Mensagem postada pelo aluno Rivaldo no Fórum Coffee Break em outubro de 2008)

Rivaldo, no entanto, vai além, trazendo, para o ambiente do curso, questões relativas à proficiência em inglês e também pessoais, quando afirma ficar confuso e ansioso devido às exigências da profissão de professor e do excesso de trabalho. A mensagem de Rivaldo também é um indicativo de que pode haver problemas no design do curso online, já que ele assegura estar receoso diante das "coisas para ler”, o que pode ser interpretado como uma quantidade excessiva de textos para leitura, selecionados no processo de design do curso.

Em resposta à postagem, dois professores e duas alunas publicam mensagem no fórum, uma mais experiente em trâmites do ambiente digital, outra iniciante como Rivaldo. Vejamos a publicação do Professor A.

Hello, dear Rivaldo! Nice to have you here!

I think that the feelings you mentioned are common among students who choose to take an online course. Believe me, for the teachers and tutors on online courses, too. Every time I start with a new group (online or not), I get a little anxious! :-)

At first, when we look at the screen, it seems to be a lot of things, but as we go on and get used to the interface and the course as a whole, things get easier and we start to look forward to getting the replies and comments and to getting in touch with the group.

Count on us for anything you need!

Love,

Professor A

(Mensagem em resposta à postagem de Rivaldo no Fórum Coffee Break, em outubro de 2008) 
Para lidar com a complexidade dos problemas trazidos para o ambiente do curso, professores e alunos interagem para colaborar e ajudar o colega participante a vencer os obstáculos e problemas relatados.

O professor A começa a mensagem recepcionando o aluno e o acolhendo. Enfatiza que ele não é o primeiro a vivenciar tal situação. O docente também compartilha experiência semelhante na condição de profissional. Por fim, ele também assegura ao aluno a solução e a superação dos obstáculos à medida que o curso vai se desenvolvendo. Sublinha que, apesar de ser o par mais experiente (VYGOTSKY, 1998) naquele contexto, compartilha de sentimentos semelhantes e garante que tais dificuldades serão superadas na interação e no desenvolvimento do curso.

Como podemos observar na mensagem a seguir, o Professor B trilha caminho semelhante ao interagir com o aluno. Acolhe-o e assegura ao aluno participante que todos os obstáculos serão vencidos com o passar do tempo, ressaltando também que os sentimentos de Rivaldo são comuns em qualquer atividade social nova.

Dear Rivaldo, nice to hear you're around.

I reckon Professor A has already said everything about your anxiety.

Mind you, dear, that whatever the new social activity is, fears, confusion, difficulties and so forth are quite common.

Take it easy, buddie. You'll see time is the best thing for you to feel more confident and comfortable afterwards.

I do hope to see you around. Many thanks!

Professor B.

(Mensagem em resposta à postagem de Rivaldo no Fórum Coffee Break, em outubro de 2008)

A colaboração não se restringe ao eixo professor-aluno-professor. Outros alunos participantes igualmente se engajam na interação e buscam colaborar com o colega para que ele vença os obstáculos por ele relatados. Assim, as alunas Moema e Eloana respondem, de igual sorte, à postagem de Rivaldo. Moema, mais experiente em cursos dessa natureza, também compartilha do posicionamento dos professores e salienta que, com o tempo e a prática, os problemas se extinguirão. Afirma, ainda, que "os bafões” serão compensados com o repensar das práticas pedagógicas dos professores participantes e com o compartilhamento das crenças pessoais de cada um. Eloana, na mesma linha, grifa problemas semelhantes e enfatiza a prática como um dos caminhos para solucionar as dúvidas e superar as dificuldades, como podemos observar a seguir. 
Hi Rivaldo,

Welcome to our forum. I'm Moema from Cuiabá, well in fact my hometown is Rio Preto but I've been here for 20 years now. I've noticed you've just joined us.

Like you, I also felt confused and insecure when I took my first on line course but with practice we overcome these feelings.

The opportunity we have to share our beliefs with peers, to rethink about our teaching practice and to practise our English, really, pays off our eventual "bafões".

So cheer up, we're all on the same boat here.

Best wishes,

Moema

Dear Rivaldo,

I know what you mean. I feel the same. Every time I come and see the forum and the agenda and the activities we have to do in the development and everything we have to do in the reflection, exercises I feel I won't be able to follow the speed of the course. It's been hard for me too. First I thought it was because it was only the beginning, but then I realize that I take too long reading the texts and reading the forums and trying to connect everything before writing my contributing. When I finally have it ready to post, It seems it is out of the context. I believe it is due to lack of practice using the environment. I don't know but I feel I'm running after the topics.

Hugs,

Eloana

(Mensagens em resposta à postagem de Rivaldo no Fórum Coffee Break, em outubro de 2008)

Tais ações não resolvem as inquietações dos alunos, nem foram planejadas no processo de design do curso. Contudo, são essenciais para o estabelecimento de relações e de uma atmosfera agradável, bem como para a consolidação da turma na qualidade de grupo. Retomando o conceito de ZDP e lembrando que "o desenvolvimento conduzido na ZDP é a crescente capacidade de se envolver em atividades volitivamente e com consciência" (NEWMAN; HOLZMAN, 2002, p. 77), percebemos que a interação gera colaboração no sentido de construção de conhecimentos necessários ao longo do curso, que vão além do linguístico ou do pedagógico. É na colaboração, e mais especificamente no compartilhamento de experiências semelhantes, que é possível desenvolver a consciência em relação às próprias ações no ambiente do curso e, assim, poder caminhar de forma mais autônoma e ter maior sucesso no curso. 
Concordamos com Nyikos e Hashimoto (1997) quando afirmam que o conhecimento é conjuntamente construído e para que a aprendizagem aconteça é necessária a interação entre, no mínimo, duas pessoas. O próximo exemplo indica que, em contextos digitais educacionais, a construção de conhecimentos pedagógicos ou linguísticos depende diretamente do domínio tecnológico. Assim, a colaboração entre os participantes, com vista à troca de informações e à busca de alternativas para solucionar os eventuais problemas, é imprescindível. A interação a seguir ocorreu entre uma aluna participante e o professor no Fórum Suporte Técnico ${ }^{5}$, alicerçada em uma postagem que buscava resolver um problema técnico com um exercício de compreensão oral.

A colaboração ocorre não só na interação registrada no fórum entre os participantes, mas também é realizada entre outros participantes externos à ferramenta. Para solucionar, ainda que parcialmente, seu problema de acesso ao material, Moema pede ajuda à colega Shirley, que envia as atividades por e-mail (ferramenta externa ao ambiente do curso) e também entra em contato com funcionário de suporte, conforme relata em uma de suas mensagens. O professor, procurando ajudar a aluna, também lança mão de outros instrumentos para que ela tenha acesso ao material e possa realizar as atividades do curso. É na colaboração no ambiente do curso e em ferramentas externas que a aluna consegue resolver suas dúvidas e dar andamento ao curso, conforme ilustram as mensagens a seguir.

Sorry, Professor A, to bother you once more, but the problem has not been solved yet, at least for me. And I can assure you that the cause has nothing to do with my computer, cause I can access all the videos with no problem at all, even other listening activities( from other sites).

As I mentioned before, when I click the file, a window opens, with the photo of the interviewee, and it reads at the top, streaming, but nothing happens. What should I do? Isn't it strange that the video is working properly?

Time for reflection and action.

Love, Moema

I cannot even open the unit 2 (development). Can you please help me solve this problem? That's the reason why I'm not taking part in the forum of discussion. What should I do?

Love, Moema

5 O Fórum Suporte Técnico destina-se à postagem de problemas de cunho técnico, tais como: problemas de acesso, incompatibilidade de ferramentas etc. 


\section{Dear Moema,}

Do you have a broad band? If your access is by dial, you may have problems opening the files. Anyhow, try this:

Open the internet explorer

Go to Ferramentas >> Opções da Internet

Exclude cookies, internet files (even the temporary ones). You do so by clicking on the buttons related to each one.

Then click OK.

Close the internet explorer

Then open it and access the course.

Please let me know if it worked!

(Mensagens trocadas entre professor e aluna no Fórum Suporte Técnico, em outubro de 2008)

O Professor A, além das orientações para a limpeza de cookies para ajudar a aluna, também solicita feedback (please let me know if it worked) para verificar se suas ações de mediação deram conta do problema enfrentado pela aluna, cuja resposta vemos na mensagem a seguir.
Dear Professor A,
I tried to solve the problem by talking to Flávio in São Paulo. He seems to be aware that the problem is with the programme cause I've being having no problems with the other activities, just Development 2. However, up-to-now no solution was made .However, I want to follow the course and in order to do so, I need at least the read- ing material. Shirley kindly sent the ones from activity one. Would you mind sending the ones from activity two? As for the listening activities what can we do?
Love, Moema
(Mensagem da aluna para o professor no Fórum Suporte Técnico, em outubro de 2008)

A aluna solicita a professor que envie o material e informa que já pediu ajuda ao funcionário de suporte técnico e à colega Shirley. O professor, então, envia o material, o que vem a se configurar como uma ação de disponibilização de material (template) por outras ferramentas não previstas no design do curso. Como a aluna não conseguiu abrir o template, a professora faz uso dos recursos "copiar" e "colar" para tornar o material acessível. 
Dear Moema,

I'm really sorry for your problem. I'll send a template by e-mail. Open it with internet explorer.

The instructions and links are there. Please, tell me if it worked, ok?

Dear Professor A,

Sorry, but it didn't work.

Love,

Moema

I'll copy and paste the texts and send them to you, ok?

Sorry it didn't work. :-(

I sent the texts by email. Did you get them?

Love,

Professor A

Dear Professor A,

Thanks a lot for your immediate reply. I opened all the material and it's working fine. Now I'll be able to do the listening exercises, too.

Love,

Moema

I'm very pleased to know that!

Work well!

(Mensagens trocadas entre professor e aluna no Fórum Suporte Técnico, em outubro de 2008) 
Esse processo é concretizado nos pedidos de feedback, nas ações de checagem e na orientação do professor, que busca, com a aluna, estratégias e instrumentos alternativos que a ajudem no processo de aprendizagem. Assim, a colaboração vai, mais uma vez, além da construção de conhecimentos pedagógicos ou linguísticos, pois engloba estratégias de aprendizagem compartilhadas no ambiente, divulgação de ferramentas alternativas e novos meios de atingir os objetivos e ter bom desempenho em cursos dessa modalidade.

Em suma, as ações de suporte construídas na colaboração e na interação entre os participantes do curso se centraram em duas esferas: a das relações e a do fazer. As ações de acolhimento, compartilhamento e asseguramento se mostraram fundamentais na consolidação do grupo e da colaboração entre os participantes, apesar de não solucionarem diretamente os problemas trazidos para o ambiente do curso. Por outro lado, as ações de orientação, checagem e feedback concretizam a atuação dos participantes do curso na busca de soluções. No entanto, todas as ações de suporte, construídas na colaboração e na interação, transpuseram a questão epistemológica, já que contribuíram para o desenvolvimento de conhecimento técnico, de navegação e de organização em um curso online, dentre outros aspectos importantes para o sucesso nessa modalidade de curso, sendo a interação e a colaboração fundamentais em tal processo, precisando ser estimulando no decorrer do curso.

\subsubsection{Terceiro estudo}

O último estudo se baseia na pesquisa de Bresolin (2011), desenvolvida com alunos do curso de Letras, habilitação Português e Inglês, de quatro universidades públicas brasileiras. Foi pensada no intuito de criar um espaço colaborativo e reflexivo em ambiente digital, para que os participantes, futuros professores de línguas, pudessem discutir vários temas ligados ao processo de ensino-aprendizagem de língua estrangeira, principalmente os assuntos relacionados com o contexto escolar público. No entanto, neste trabalho, focalizamos, nos dados, como as escolhas linguísticas dos participantes podem criar contextos mais apropriados para prática de processos colaborativos. Ao observar exemplos empíricos, podemos trazer maneiras mais operacionais na sala de aula que estimulem, por meio da linguagem, momentos mais colaborativos.

O blog, ambiente gerador da pesquisa, foi coordenado por um dos autores deste artigo, em parceria com professores universitários que ministravam disciplinas relacionadas com a língua inglesa e/ou com o ensino de língua estrangeira nas respectivas turmas. As interações digitais começaram a ocorrer em maio de 2010 e foram efetivamente desenvolvidas, com os colegas, de junho a dezembro do mesmo ano. Para este trabalho, desdobraremos alguns exemplos ilustrativos dos processos de colaboração entre os participantes. 
Nos exemplos a seguir, os comentários, além de trazerem as descrições de fatos vivenciados pelos participantes, trazem um tom de expectativa relacionada com o projeto do blog, com traços de manifestação entusiasta dos participantes e inúmeras tentativas para que o grupo comece a se conhecer melhor e a se estabelecer nesse novo ambiente.

Aluna: Hello, we are Heloisa and Carolina we study at UFMT campus Ron-
donópolis. We are happy to share our opinion in this blog. We hope to learn
and share our knowledge about English teaching and the texts that we see at
University. Heloísa has a blog that talks about herself, if you want to know
more information about Heloisa come to Heloblog.blogspot.com

See you guys,

Have a Nice week (-) (agosto de 2010).

Nesse exemplo, há alguns aspectos que nos ajudam a pensar no processo de colaboração. A aluna se vale do pronome "we" como identificador do grupo ao qual pertence. Por meio desse recurso, constrói-se, nesse instante, uma identidade coletiva. A utilização do sintagma hope to learn and share, além do léxico happy e do endereço para o blog de um dos participantes, pode ser entendida como características que sinalizam o desejo de que se instaure uma comunidade colaborativa, uma vez que ela depende, justamente, dessa identificação ou desse sentimento de grupo. Da mesma forma, as saudações e o encerramento das mensagens parecem ter a finalidade de criar laços de aproximação entre quem envia uma mensagem e quem a recebe.

Essa sinalização é, por sua vez, de suma importância, visto que pode passar a produzir caminhos para uma cultura colaborativa de aprendizagem, prática essa sugerida por Nyikos e Hashimoto (1997) e Figueiredo (2006) como pilar essencial no processo de formação inicial e continuada, não só dos professores mas de qualquer profissional. Assim, essa postura colaborativa faz parte de um processo de compartilhamento das responsabilidades acerca do processo de ensino-aprendizagem de todo grupo.

Pelo que se percebeu, ao longo da pesquisa, os participantes pareciam se envolver mais colaborativamente no blog. Talvez, uma das razões seja o fato de que a produção escrita dos alunos se apresentava dentro de um contexto mais significativo com leitores que debatiam e questionavam os tópicos que se mostravam na interação em curso. Entendemos que, quanto mais os participantes de uma sala de aula digital vivenciarem experiências compartilhadas, expondo suas emoções e comportamentos que os caracterizam como sujeitos em um ambiente de aprendizagem, mais possiblidade poderá haver de uma cultura colaborativa.

Essas emoções auxiliam no engajamento em diálogos significativos na experiência educacional, refletindo condições necessárias para processos colabora- 
tivos. Dessa forma, pode ser criado um clima de aceitação entre os participantes que os auxiliam na construção de ações de reconhecimento, cumplicidade e de prontidão para as respostas dos outros participantes. Contudo, deve-se asseverar que somente a exposição online não garante esse contexto. De que forma o blog, então, estimula este processo? No caso do blog desenvolvido nesta pesquisa, houve a tentativa de agregar e consolidar um grupo de debate, o que, de certa forma, pode ter criado essa sensação de dinamismo, colaboração e crescimento profissional e pessoal. Nos próximos exemplos, percebemos que também há traços de colaboração neste sentido.

\begin{abstract}
Aluna: Hi, my name is Carolina but you can call me Carol. I'm from Campo Mourão-PR. I'm 25 years old. I'm a student at FECILCAM. Letters Course is the second course that I started, but this one I really want to finish. I'm very happy with this blog. It's a very nice way to learn more and change information. NICE TO MEET YOU. (Setembro, 2010)
\end{abstract}

\title{
Professora: Hi dear,
}

Thanks a lot!!! The blog has been a great success because most of the students are very concerned about how English classes happen and how they can be improved. When my professor XXXX said to me we were going to participate I became so happy. I'm from Paraná and I graduated at UCP, in Pitanga, that is too near Campo Mourão. I also had classes with Dr. Monica XXX (in fact she was my supervisor) and with Dr. xxxxxx, that taught me a lot about Sociointeractionism and how to use song in the English classes.

I believe the blog is gonna be a very important way to share ideas, to know different people, read about a sort of opinions and discuss important topics for the students that are still in the university.

So, welcome again XXXX and everybody from FECILCAM! (Setembro 2010)

Nesses exemplos, observamos o uso das expressões NICE TO MEET YOU e welcome que geram um ambiente mais aconchegante, somado à informalidade que a aluna solicita que seja tratada no grupo - you can call me Carol -, acrescido também dos adjetivos happy e nice. A professora, por sua vez, na tentativa de criar um vínculo com a estudante, procura trazer suas experiências no período da graduação na mesma instituição da qual a aluna fazia parte. Por último, a professora procura enfatizar a importância do blog como um veículo de aprendizagem em que os participantes possam se sentir integrados a um grupo para refletir com sua comunidade sobre diferentes assuntos inerentes à carreira do professor de língua inglesa.

Acreditamos que a criação de vínculos de colaboração tenha sido um dos fatores cruciais para que o projeto fosse bem-sucedido. Dentre as maiores con- 
tribuições desse processo colaborativo, destacamos a mobilização e a valorização do projeto, como parte integrante da aprendizagem curricular. Em outras palavras, os alunos viam o blog como um veículo de desenvolvimento linguístico e de formação docente que se estendeu do meramente presencial e passou a se dar também virtualmente.

Outro ponto de suma importância foram os comentários produzidos pelos professores universitários participantes da pesquisa. Como pares mais competentes (VYGOTSKY, 1998), mobilizaram questões teóricas, profissionais e de ordem linguística, como é ilustrado no seguinte excerto:

\begin{abstract}
Aluno: I completely agree with those previous commentaries... As soon as I read those I remembered a teacher of mine who said once to my class that 'students are not all the same to fit in the same bottle'. This bottle refers to the same way teachers use to teach their students by following the same curriculum.

Indeed, I reckon a teacher 'must' check if in a way (individually or in relation to the classes in general) he/she is using this bottle. To my mind, it's really important to be a reflexive teacher. However, I also think teachers can't just throw the previous schedule away, but observe what can be preservated and what should be replaced for something else. In a brief, the do's of acting like this are the result of a good teaching practice while the don'ts are so expansive that we can't simply consider as a bad result.
\end{abstract}

Em relação à colaboração, possíveis marcas podem ser verificadas já no início do comentário, no momento em que o aluno afirma concordar com outros participantes em postagem anteriores - I completely agree with those previous comentaries. Esse comentário revela que questões significativas foram apresentadas e que favoreceram uma reflexão sobre o papel de um professor ante o uso de métodos/metodologias: As soon as I read those I remembered a teacher of mine who said once to my class that students are not all the same to fit in the same bottle.

Em resumo, nesta seção, buscamos trazer exemplos que tipificam linguisticamente amostra de processos de colaboração com a finalidade de entender os possíveis tipos de interações colaborativas presentes em processos de ensino e aprendizagem de línguas.

\title{
11.4 CONSIDERAC̣ÕES FINAIS
}

Neste capítulo, procuramos, por meio de um olhar interpretativo, discutir os processos de construção colaborativa em três diferentes contextos. Por meio da análise dos dados, percebemos que as interações que emergiram podem ser interpretadas como possíveis marcas de desenvolvimento de processos colaborativos.

No estudo 1, pudemos observar que projetos telecolaborativos podem ser uma forma alternativa de ensino de línguas em contextos tecnológicos. Os inte- 
ragentes puderam, juntos, se auxiliar na aprendizagem das línguas que estavam aprendendo por meio de interações significativas proporcionadas por meio de um aplicativo computacional de mensageira instantânea: o Openmeetings. Verificamos também que a língua inglesa serviu aos interagentes como um scaffolding para a aprendizagem da língua portuguesa e da língua alemã.

No estudo 2, constatamos que a aprendizagem colaborativa não se limita à apreensão e à aquisição de conteúdo linguístico e pedagógico, mostrando-se, em contexto de cursos online, fundamental para o desenvolvimento de conhecimentos técnicos e de habilidades imprescindíveis para os trâmites do ambiente digital, o que contribui para o bom desempenho e sucesso no curso. Nesse sentido, a colaboração se materializa, principalmente, em ações de suporte de compartilhamento, acolhimento e asseguramento. Tais ações nem sempre resultam na solução das dificuldades e dos problemas relatados no ambiente do curso, mas se mostraram essenciais para o estabelecimento de relações e consolidação do grupo.

No estudo 3, demonstramos que o blog se caracterizou como uma rede social que tendeu a reimprimir uma cultura mais colaborativa. Contudo, não podemos negar que, vez por outra, podem se estabelecer processos individualistas com pouca troca entre os participantes. Cabe à universidade, aos grupos formadores e aos próprios alunos em formação considerar a complexidade que o tema enfeixa, de modo a propor o trabalho de alteridade como uma prática contínua em suas vivências profissionais.

Finalmente, com base na revisão desses três estudos, podemos concluir que a colaboração no meio virtual se configura, pois, como uma abordagem extremamente importante para o processo de aprendizagem por proporcionar interações significativas, por intermédio das quais os interagentes podem auxiliar uns aos outros, esclarecer dúvidas e encontrar caminhos para a realização das atividades.

Numa perspectiva vygotskiana, um ambiente mais interativo e colaborativo tem o potencial de favorecer a aprendizagem, como é ilustrado nos estudos de Silva (2012), Ribeiro (2010) e Brezolin (2011). Dessa forma, a telecolaboração deve ser vista como uma abordagem extremamente importante para o processo de aprendizagem de línguas e de formação de professores por proporcionar interações significativas. Graças a elas, os interagentes podem ter um papel mais ativo como aprendizes de uma língua estrangeira e no desenvolvimento de habilidades importantes para sua formação em contextos digitais.

\section{REFERÊNCIAS}

BEDRAN, P. F; SALOMÃO, A. C. B. Interação de crenças em contexto virtual de aprendizagem de língua. Revista Brasileira de Linguística Aplicada, Belo Horizonte, v. 13, n. 13, p. 789-814, 2013. 
BRESOLIN, A. R. O professor de línguas em formação: uma experiência reflexiva com blog. 2011. 146 f. Dissertação (Mestrado em Estudos de Linguagem) - Universidade Federal de Mato Grosso, Cuiabá, 2011.

BRUFFEE, K. A. Collaborative learning: higher education, interdependence, and the authority of knowledge. London: The Johns Hopkins University Press, 1999.

CARELLI, I. M. Estudar online: análise de um curso de professores de professores de inglês na perspectiva da teoria da atividade. 2003. 223f. Tese (Doutorado em Linguística Aplicada e Estudos da Linguagem) - Pontifícia Universidade Católica de São Paulo, São Paulo, 2003

COELHO, E. Cooperative learning: foundation for a communicative curriculum. In: KESSLER, C. (Ed.). Cooperative language learning: a teacher's resource book. New Jersey: Prentice Hall Regents, 1992. p. 31-49.

DEBSKI, R. Project-oriented learning with technology: implementation and directions of enquiry. In: CARMAGNANI, A. M. G.; GRIGOLETTO. M. (Org.). Inglês como língua estrangeira: identidade, práticas e textualidade = English as a foreign language: identity, practices and textuality. São Paulo: Humanitas, 2001. p. 195-209.

DILLENBOURG, P. What do you mean by collaborative learning. In: DILLENBOURG, P. (Ed.). Collaborative learning: cognitive and computational approaches. Oxford: Elsevier. 1999. p. 1-19. Disponível em: <http://tecfa.unige.ch/tecfa/publicat/dil-papers-2/ Dil.7.1.14.pdf>. Acesso em: 10 mar. 2004.

DONALDSON, S. Intensive ESL courses at CALS. Carleton Papers in Applied Language Studies, v. 7, p. 6-25, 1990.

DONATO, R. Aspects of collaboration in pedagogical discourse. Annual Review of Applied Linguistics, v. 24, p. 284-302, 2004.

FIGUEIREDO, F. J. Q. de. Semeando a interação: a revisão dialógica de textos escritos em língua estrangeira. Goiânia: Ed. da UFG, 2005.

FIGUEIREDO, F. J. Q. de. (Org.). A aprendizagem colaborativa de linguas. Goiânia: Ed. da UFG, 2006.

FIGUEIREDO, F. J. Q. de.; GONÇALVES, R. M. Você já blogou hoje? Estudo sobre o uso de blogs nas aulas de língua inglesa. In: JESUS, D. M.; MACIEL, R. F. (Org.). Olhares sobre tecnologias digitais: linguagens, ensino, formação e prática docente. Campinas: Pontes, 2015. p. 325-353.

FIGUEIREDO, F. J. Q. de.; SILVA, S. V. A colaboração no ensino-aprendizagem de línguas em contextos tecnológicos: uma análise das interações entre aprendizes brasileiros e alemães. In: SIMÕES, D. M. P.; FIGUEIREDO, F. J. Q. de. (Org.). Metodologias em/de linguística aplicada para ensino e aprendizagem de línguas. Campinas: Pontes, 2014. p. 73-91.

. Telecolaboração entre alunos brasileiros e alemães: uma análise do uso de ferramentas mediacionais. In: OSÓRIO, P.; BERTINETTI, F. de S. (Org.). Teorias e usos linguísticos: aplicações ao português língua não materna. Lisboa: Lidel, 2015. p. 183-206. GONÇALVES, R. M. Você já blogou hoje? Um estudo de caso sobre o uso de blogs na aula de língua inglesa. 2009. 167 f. Dissertação (Mestrado em Letras e Linguística) Universidade Federal de Goiás, Goiânia, 2009. 
JESUS, D. M. Reculturação, reestruturação e reorganização temporal de professores no ambiente digital. 2007. 221 f. Tese (Doutorado em Linguística Aplicada) - Pontifícia Universidade Católica de São Paulo, São Paulo, 2007.

. Navegando pela aprendizagem de professores de lingua inglesa em cursos online. Cuiabá: EdUFMT, 2014.

JESUS, D. M.; MACIEL, R. F. (Org.). Olhares sobre tecnologias digitais: linguagens, ensino, formação e prática docente. Campinas: Pontes, 2015.

LEFFA, V. J. Interação simulada: um estudo da transposição da sala de aula para o ambiente virtual. In: LEFFA, V. J. (Org.). A interação na aprendizagem das línguas. Pelotas: EDUCAT, 2003. p. 175-218.

LELOUP, J. W.; PONTERIO, R. Tele-collaborative projects: Monsters.com? Language Learning \& Technology, v. 7, n. 2, p. 6-11, 2003. Disponível em: <http://lt.msu.edu/ vol7num2/net/default.html>. Acesso em: 21 maio 2004.

LIANG, X.; MOHAN, B. A.; EARLY, M. Issues of cooperative learning in ESL classes: a literature review. TESL Canada Journal, v. 15, n. 2, p. 13-23, 1998.

LIMA, M. dos S. Collaborative tasks and learning occasions in English as a foreign language. Revista Brasileira de Linguística Aplicada, v. 11, n. 4, p. 837-852, 2011.

MARTINO, L. M. S. Teoria das midias digitais: linguagens, ambientes, redes.

Petrópolis; Vozes, 2015.

NUNES, B. R. S. S. Leitura em língua inglesa: a resolução colaborativa de exercícios de compreensão textual. 2002. Dissertação (Mestrado em Letras e Linguística) - Faculdade de Letras, Universidade Federal de Goiás, Goiânia, 2002.

NEWMAN, F.; HOLZMAN, L. Lev Vygostsky cientista revolucionário. Tradução de Marcos Bagno. São Paulo: Edições Loyola, 2002.

NYIKOS, M.; HASHIMOTO, R. Constructivist theory applied to collaborative learning in teacher education: in search of ZPD. The Modern Language Journal, v. 81, n. 4, p. 506-517, 1997.

PAIVA, V. L. M. de O. e. A www e o ensino de inglês. Revista Brasileira de Linguística Aplicada, v. 1, n. 1, p. 93-116, 2001a.

PAIVA, V. L. M. de O. e. (Org.). Interação e aprendizagem em ambiente virtual. Belo Horizonte: UFMG, 2001b.

RIBEIRO, A. S. M. Design e suporte em contexto de curso online: um estudo sob a perspectiva da Teoria da Atividade. 2010. 229 f. Tese (Doutorado em Linguística Aplicada e Estudos da Linguagem) - Pontifícia Universidade Católica de São Paulo, São Paulo, 2010.

SILVA, S. V. da. O processo ensino-aprendizagem de linguas em teletandem: um estudo na área de Turismo. 2012. 293 f. Tese (Doutorado em Letras e Linguística) - Faculdade de Letras, Universidade Federal de Goiás, Goiânia, 2012.

SILVA, S. V. da.; FIGUEIREDO, F. J. Q. de. O processo de ensino e aprendizagem de inglês e português em teletandem: foco no uso de recursos mediacionais. In: CONGRESSO INTERNACIONAL DA ABRAPUI, 3., 2012, Florianópolis. Anais... Florianópolis: UFSC, 2012. p. 1-15. 
SOUZA, R. R. Aprendizagem colaborativa em comunidades virtuais: o caso das listas de discussão. In: COSCARELLI, C. V. (Org.). Novas tecnologias, novos textos, novas formas de pensar. Belo Horizonte: Autêntica, 2002. p. 95-109.

SOUZA, R. A. Aprendizagem de Linguas em Tandem - Estudo da telecolaboração através da comunicação mediada pelo computador. 2003. Tese (Doutorado em Letras) Universidade Federal de Minas Gerais, Belo Horizonte, 2003.

SWAIN, M. The output hypothesis and beyond: mediating acquisition through collaborative dialogue. In: LANTOLF, J. P. (Ed.). Sociocultural theory and second language learning. Hong Kong: Oxford University Press, 2000. p. 97-114.

TELLES, J. A. (Org.). Teletandem: um contexto virtual, autônomo e colaborativo para aprendizagem de línguas estrangeiras no século XXI. Campinas: Pontes Editores, 2009. TSUI, A. B. M. Introducing Classroom Interaction. London: Penguin, 1995.

VASSALLO, M. L.; TELLES, J. A. Foreign language learning in-tandem: Teletandem as an alternative proposal in CALLT. The ESPecialist, v. 27, n. 2, p. 189-212, 2006. . ensino e aprendizagem de línguas em tandem: princípios teóricos e perspectivas de pesquisa. In: TELLES, J. A. (Org.). Teletandem: um contexto virtual, autônomo e colaborativo para aprendizagem de línguas estrangeiras no século XXI. Campinas: Pontes Editores, 2009. p. 21-42.

VYGOTSKY, L. S. The genesis of higher mental functions. In: WERTSCH, J. V. (Ed.). The concept of activity in Soviet psychology. New York: M.E. Sharpe, 1981. p.144-188. . A formação social da mente: o desenvolvimento dos processos psicológicos. São Paulo: Martins Fontes, 1998.

WARSCHAUER, M. Computer-mediated collaborative learning: theory and practice. The Modern Language Journal, v. 81, n. 3, p. 470-481, 1997.

WOOD, D.; BRUNER, J.; ROSS, G. The role of tutoring in problem solving. Journal of Child Psychology and Psychiatry, v. 17, p. 89-100, 1976. 
